\title{
Pelargonien im Palmengarten
}

\author{
Hilke Steinecke
}

\begin{abstract}
In connection with the more general theme South Africa in 2006, the Palmengarten displayed an exhibition devoted to Pelargonium. Species and cultivars were exhibited, along with information on biological, horticultural and cultural aspects of the genus Pelargonium and on the medical use of several species. Some of these aspects are summarized in this paper.

\section{Zusammenfassung}

Im Rahmen des Südafrika-Schwerpunktes im Jahr 2006 zeigte der Palmengarten eine Pelargonien-Ausstellung. Ergänzend zur floristischen Gestaltung mit vielen Wildarten und gärtnerischen Sorten gab es Informationen zur Biologie, Kulturgeschichte, Pflege der Pelargonien sowie ihrer Nutzung als Medizinalpflanzen. Einige dieser Informationen werden im Beitrag vorgestellt.
\end{abstract}

\section{Südafrika und Pelargonien im Palmen- garten}

Das Veranstaltungsprogramm 2006 des Palmengartens stand unter dem Motto „Südafrika“. Verschiedene Ausstellungen, Führungen und andere Aktionen widmeten sich der südlichen Spitze des „Schwarzen Kontinents“. Auftakt des Südafrika-Jahres bildete eine Pelargonien-Ausstellung im Mai 2006. Ein Themenschwerpunkt waren dabei die medizinisch genutzten Pelargonien, weshalb auch ein Apothekerschrank ein Beet zierte. Da sich die Pelargonien gut in der Ausstellungsgalerie hielten und immer wieder neue Blüten bildeten, blieb ein Teil der Pflanzen auch nach dem offiziellem Ausstellungsende erhalten und bereicherte die Präsentation anderer Zierpflanzen aus Südafrika. Als Ergänzung der Ausstellung schmückten viele verschiedene Duftpelargonien wie in jedem Jahr die Beete vor dem Haus Leonhardsbrunn. Im heißen Juni und Juli verströmten die aromatischen Pflanzen einen besonders intensiven Duft. Die Mutterpflanzen unserer Pelargonien sind in der Gärtnerei des Palmengartens untergebracht, für die Schaubeete wurden Jungpflanzen aus Stecklingen herangezogen.

$\mathrm{Da}$ immer wieder nach einer schriftlichen Version der in der Ausstellung gelieferten Informationen rund um die Pelargonien gefragt wurde, sollen hier die Texte der Ausstellungstafeln in leicht abgeänderter Form, ergänzt durch Informationen aus dem Botanischen Garten der Universität Wien, veröffentlicht werden. Als wichtige Quellen zu diesem Artikel dienten die Publikationen von van Der WALt (1979), Gugenham (1981), Yeo (1985) und Wiegele (2000). Einige wichtige Hinweise stammten von Frank Schumacher.

\section{Kleines Familienporträt der Storchschna- belgewächse (Geraniaceae)}

Storchschnabelgewächse umfassen um die 800 Arten und 11 Gattungen. Pelargonium ist mit über 250 Arten die größte Gattung. Weitere interessante Gattungen sind z.B. Geranium, Erodium, Monsonia und Sarcocaulon. Geraniaceae sind weltweit verbreitet und kommen bevorzugt in gemäßigten und subtropischen Gebieten der Nord- und Südhalbkugel vor. Blätter und Stängel sind häufig mit Drüsenhaaren versehen. Die Blüten vieler Storchschnabelgewächse sind auffällig gefärbt und haben jeweils 5 Kelch- und Kronblätter. Der Fruchtknoten ist oberständig und besteht meist aus 5 verwachsenen Fruchtblättern. Die Früchte sind oft lang und schmal und erinnern an einen Vogelschnabel. Es handelt sich meist um Spaltfrüchte, bei denen sich die Außenwände der Fruchtblätter von einer Mittelsäule ablösen. Der obere grannenartige Teil ist im feuchten Zustand gestreckt und krümmt oder spiralisiert sich korkenzieherartig unter trockenen Bedingungen.

In der einheimischen Wildflora sind aus der Familie die Gattungen Geranium (Storchschnabel) und Erodium (Reiherschnabel) vertreten. Storchschnabel-Arten besiedeln verschiedene Standorte. So kommt beispielsweise der Stinkende Storchschnabel ( $G$. robertianum) häufig 

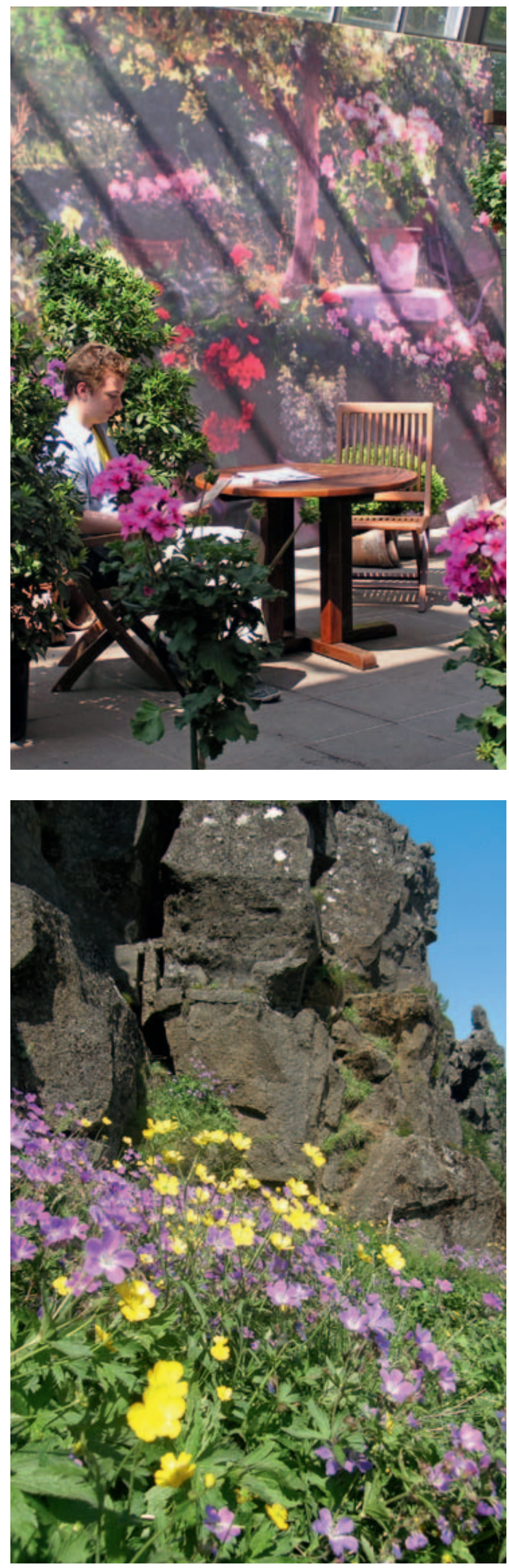

an nährstoffreichen Weg- und Waldrändern vor, während Wiesen- und Wald-Storchschnabel (G. pratense und G. sylvaticum) auf fetten und feuchten Wiesen gedeihen. Der Blutrote Storchschnabel ( $G$. sanguineum) bevorzugt Trockensäume und wärmeliebende Gebüsche und ist auch eine beliebte Gartenstaude.

Viele verschiedene Geranium-Arten, -Sorten und -Hybriden aus aller Welt werden wegen ihres hohen Zierwertes in Gärten kultiviert. Der Madeira-Storchschnabel bildet kleine Stämme und muss bei uns im (Gewächs-)haus überwintert werden.

Der dem Storchschnabel ähnelnde Reiherschnabel unterscheidet sich von diesem vor allem durch seine gefiederten Blätter, während Geranium rundliche bis handförmig geteilte Blätter hat. Die Fruchtschnäbel von Geranium sind zur Reifezeit bogig aufwärts gekrümmt, die Teilfrüchte von Erodium rollen sich dagegen spiralig ein. Durch wiederholtes Spiralisieren und Entspiralisieren verankern sich die Bohrfrüchte im Boden. Befestigt man diese kleinen „Drillbohrer“ an einer Pappscheibe, kann man sich ein einfaches Hygrometer basteln. In Abhängigkeit von der Luftfeuchtigkeit sind dann die Grannen unterschiedlich stark gedreht und die Grannenspitze zeigt in verschiedene Richtungen. Der meist höchstens zehn Zentimeter große Gemeine Reiherschnabel (Erodium cicutarium) ist eine ein- bis zweijährige Art in Weinbergen, auf Brachen und Sandtrockenrasen oder an Wegrändern.

Die 14 Arten der Gattung Sarcocaulon stammen wie die meisten Pelargonien aus Südafrika. Sarcocaulon wird auch als Buschmannskerze bezeichnet, da ihre dicken Stängel eine wachsartige, brennbare Beschichtung haben. Wenn die Stängel angezündet werden, brennen sie wie Fackeln. Diese Eigenschaft nutzten die Einheimischen, in dem sie Spross-Stücke als Kerzen oder Fackeln verwendeten.

Abb. 1 (oben): In der Pelargonienausstellung des Palmengartens.

Abb. 2 (unten): Geranium sylvaticum ist in Deutschland heimisch, ist aber auch in ganz Nordeuropa weit verbreitet; hier Exemplare aus Island (Thingvellir). 


\section{Biologie, Verbreitung und Kulturge- schichte von Pelargonium}

Die meisten Pelargonien-Arten kommen in Südafrika vor. Da fast alle Arten auf der Südhalbkugel beheimatet sind, werden sie gelegentlich auch als die Sterne des Südens bezeichnet. Die meisten bevorzugen Gebiete im Südwesten und im Süden Südafrikas. Einige Arten sind aber auch in der Lage, in diesen Gebieten wie der Namibwüste, der Karoo oder im Hochveld zu wachsen. Arten extrem trocken-heißer Gebiete sind durch wasserspeichernde Wurzeln oder Sprossachsen, harte Blätter oder das Abwerfen von Blättern an diese besonderen Bedingungen angepasst.

In den Trockengebieten sind kleinklimatisch bevorzugte Standorte (z. B. im Schatten von Sträuchern und Felsen, in der Nähe von Bächen, an der Regenseite von Gebirgen) günstige Wuchsorte für Pelargonien. In Gebieten mit Mittelmeerklima sind die Niederschlagsverhältnisse und die Luftfeuchte ausreichend und die Temperaturen gemäßigt, sodass hier die meisten Pelargonien-Arten anzutreffen sind. Während in Teilen der heißen trockenen Namib 13 Arten wachsen (Nebelgebiete, Westhänge der Küstengebirge), beträgt die Artenzahl in der Karoo und im randtropischen Bereich bis zu acht. In der Kalahari, im Hochveld und in Zentral- und NO-Südafrika gibt es keine oder nur einzelne Pelargonium-Arten, da es für sie dort zu heiß und trocken ist.

Weiter nördlich kommen in Afrika nur wenige Pelargonien (18 Arten) im montanen und küstennahen Osten bis nach Äthiopien und Madagaskar vor. Im Süden Australiens und auf Neuseeland wachsen acht Arten, von denen die meisten als einjährige oder kurzlebige und unauffällige Kräuter auf Ruderalflächen zu finden sind. Im Südatlantik kommen auf den isolierten Inseln Tristan da Cunha und St. Helena je eine Art vor. Auf St. Helena wächst die endemische

Abb. 3 (oben): Pyrenäen-Reiherschnabel (Erodium manescavi) lässt sich bei uns an sonnigen und trockenen Standorten gut als Zierpflanze halten.

Abb. 4 (unten): Brennende Rinde einer Buschmannskerze.
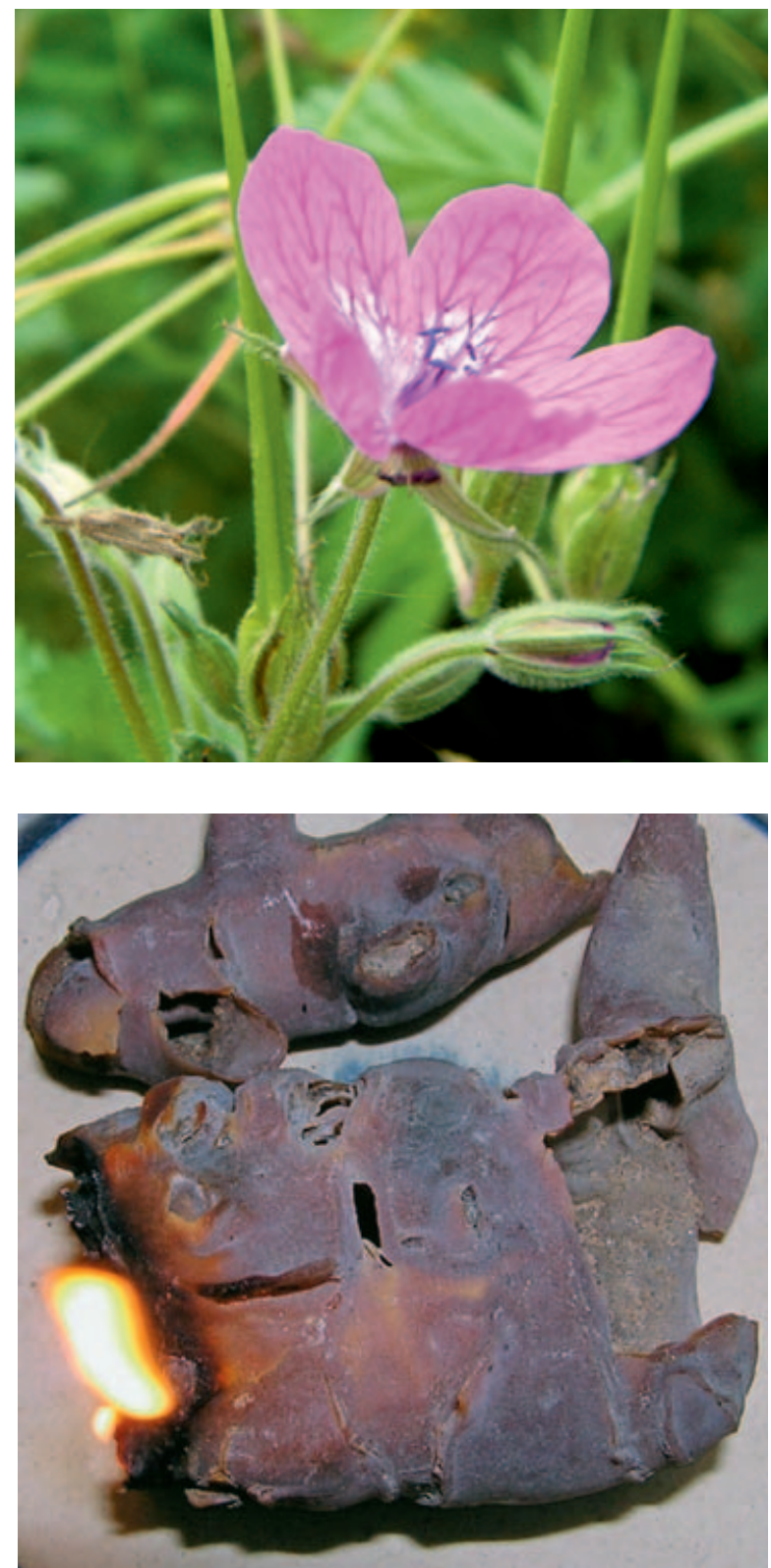

P. cotyledonis. Nur zwei Arten wachsen nördlich von Afrika. Pelargonium endlicherianum findet man in Ostanatolien und P. quercetorum in den Bergen des Nordiraks. Beide Arten haben sehr attraktive Blüten und sind in milden Gebieten Mitteleuropas mit guter Drainage und Schutz vor kalter Nässe von oben sogar winterhart.

Die ersten Pelargonien, die aus Südafrika nach Europa gelangten, wurden zunächst der Gattung Geranium zugeordnet. Der Name leitet sich von geranos (= Kranich) ab und bezieht sich auf die lang geschnäbelte Frucht, die an einen Vogelkopf erinnert. Im Jahr 1789 wurden sie dann von dem französischen Botaniker L'Héritier de Brutelle in die Gattung 


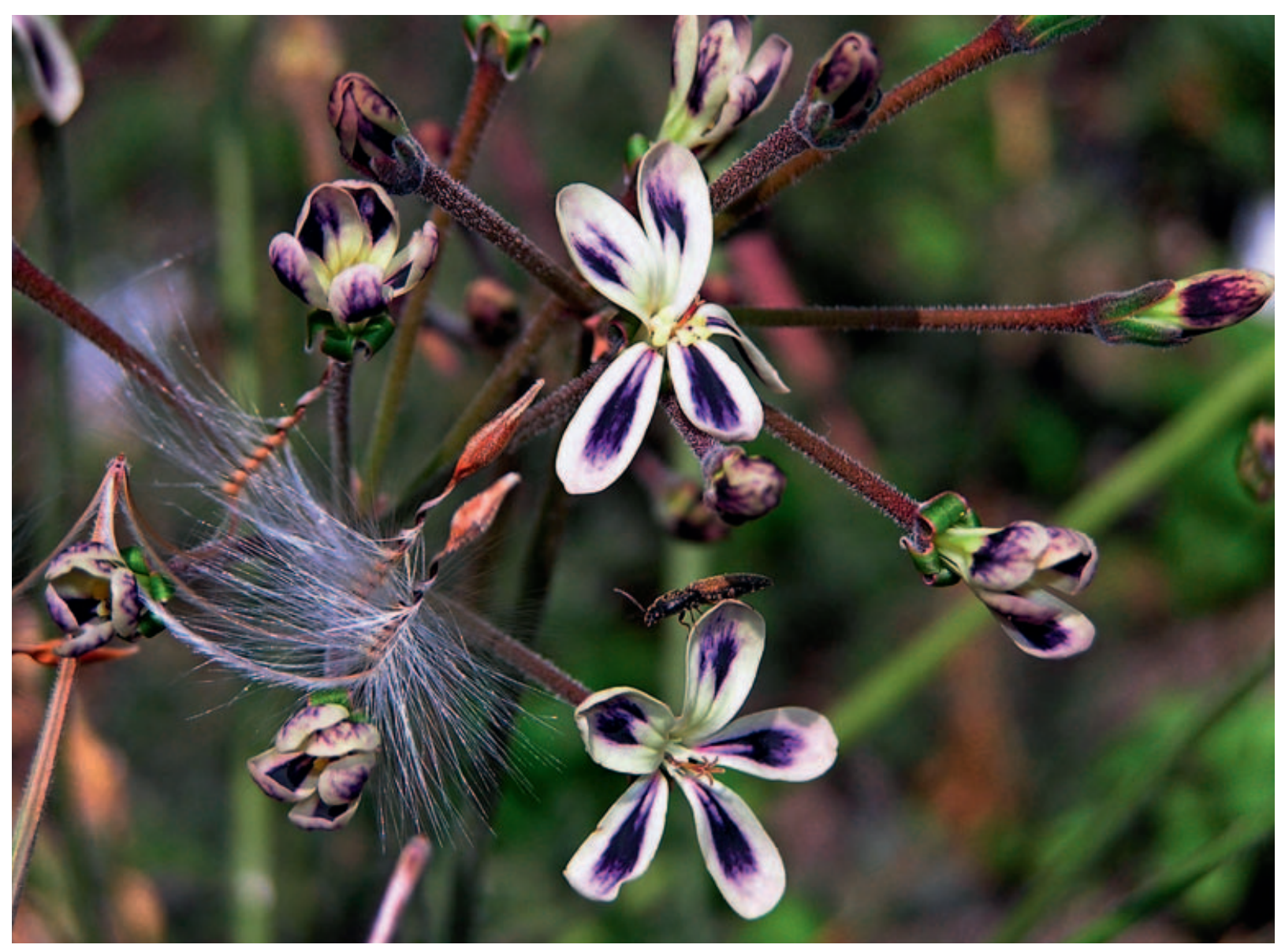

Pelargonium gestellt. Die nach wie vor häufig verwendete deutsche Bezeichnung Geranie kann zu Missverständnissen führen, denn unter Geranium (Storchschnabel) wird heute eine über 200 Arten umfassende Gattung verstanden, die sich in mehreren Merkmalen von den Pelargonium-Arten unterscheidet. Irritierenderweise leitet sich der Name Pelargonium von pelargos (=Storch) ab, wobei die deutsche Bezeichnung Storchschnabel wiederum für $G e-$ ranium vergeben ist.

Geranium-Arten entwickeln radiärsymmetrische Blüten ohne Sporn. Alle 10 Staubgefäße sind fruchtbar. Pelargonien dagegen bilden Blüten mit zwei Symmetriehälften. Von den 10 Staubblättern sind höchstens 7 fruchtbar. Die Grannen der schnabelförmigen Spaltfrüchte beider Gattungen rollen sich beim Eintrocknen korkenzieherartig zusammen.

Pelargonium triste mit ihren kleinen, schwarzpurpur und grün gefärbten Blüten wurde 1621 als erste Pelargonie aus Südafrika nach Europa gebracht. Vom 17. bis 19. Jh., als das Kapland niederländische Kolonie war, kamen zahlreiche Pelargonien-Arten zunächst nach Holland. Die trockenheitstoleranten Pflanzen ließen sich ohne größere Verluste zusammen mit anderer Schiffsladung transportieren. Bald etablierten sie sich aber auch in anderen Teilen Europas. Zu den ersten wichtigsten kultivierten Arten zählen $P$. zonale, $P$. peltatum, $P$. inquinans und $P$. grandiflorum. Durch Kreuzung und Auslese entstanden innerhalb weniger Jahrzehnte zahlreiche Sorten. Im Jahr 1782 wuchsen im SenckenberGGarten in Frankfurt bereits 18 Arten und Kultivare. Im 18. Jh. züchteten besonders die Engländer großblumige Sorten (s.g. Grandiflorum-Hybriden), die als Edelpelargonien oder Englische Pelargonien bezeichnet wurden und an deren Entstehung die Baum-Pelargonie (P. cucullatum) aus dem südafrikanischen Fynbos maßgelich beteiligt ist. Im 19. Jh. selektier-

Abb. 5: Pelargonium triste. Gut erkennbar sind die im trockenen Zustand korkenzieherartig spiralisierten Teilfrüchte. 
ten deutsche und französische Züchter die so genannten Deutschen und Französischen Pelargonien. Heute sind auch viele in den USA entstandene Sorten auf dem Markt. Es gibt aber auch interessante und erfolgreiche Neuzüchtungen aus Deutschland, z. B. von dem Familienunternehmen ELSNER pac Jungpflanzen.

$\mathrm{Zu}$ den bekanntesten und am meisten verwendeten Pelargonien gehören die ZonaleHybriden, die aus Kreuzungen zwischen der Gürtel-Pelargonie ( $P$. zonale) und der Scharlach-Pelargonie (P. inquinans) entstanden sind. Da diese Hybriden aufrecht wachsen, werden sie auch als Steh-Pelargonien bezeichnet. Die hängenden Efeu-Pelargonien dagegen sind aus Kreuzungen von $P$. peltatum mit $P$. lateripes hervorgegangen. Seit gut 100 Jahren gibt es auch Hybriden aus Gürtel- und Efeu-Pelargonien. Franzosen und Engländer waren die ersten Züchter dieser Hybriden.

Selten kultiviert und weniger bekannt sind sukkulente Arten wie beispielsweise P. crassicaule oder P. alternans mit dickem, kurzem Stamm. Solche Arten besonders trockener Standorte haben im Vergleich zur Gürtel-Pelargonie meist relativ kleine, tief eingeschnittene Blätter mit oft sehr schmalen Blattzipfeln.

\section{Pelargonien als Zierpflanzen}

Pelargonien gehören in Mitteleuropa zu den beliebtesten Balkon- und Fensterbrettpflanzen. Besonders prächtig gedeihen sie auf den in den Alpenländern vielerorts üppig bepflanzten Balkonen, häufig in Kombination mit verschiedenen anderen Sommerblumen wie Petunien oder Zweizahn (Bidens). Im Frühjahr wird in vielen Gegenden auf Geranienmärkten eine Vielzahl an Kulturformen angeboten. Von der Vielfalt der attraktiven Pelargonien (Arten sowie Sorten) konnte man sich in der Ausstellung einen guten Eindruck verschaffen.

Pelargonien werden gärtnerisch in verschiedene Gruppen eingeteilt:

Abb. 6 (oben): Gürtel-Pelargonie 'Turkish Delight'.

Abb. 7 (unten): Pelargonium crassicaule, eine sukkulente Art.
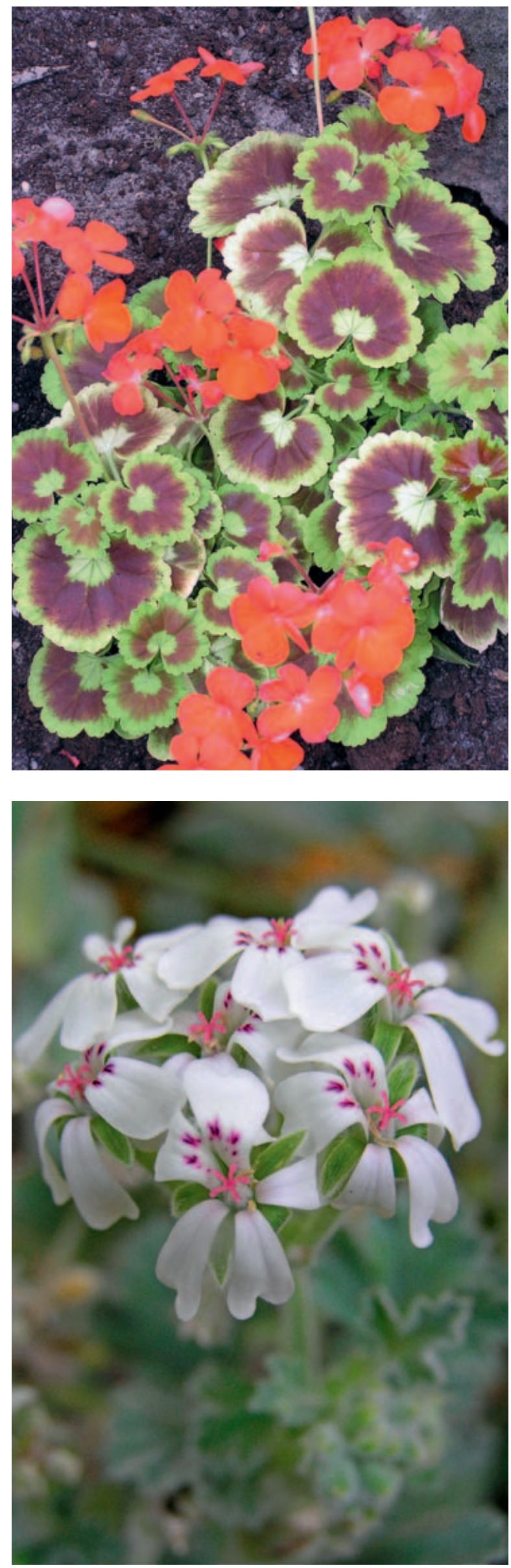

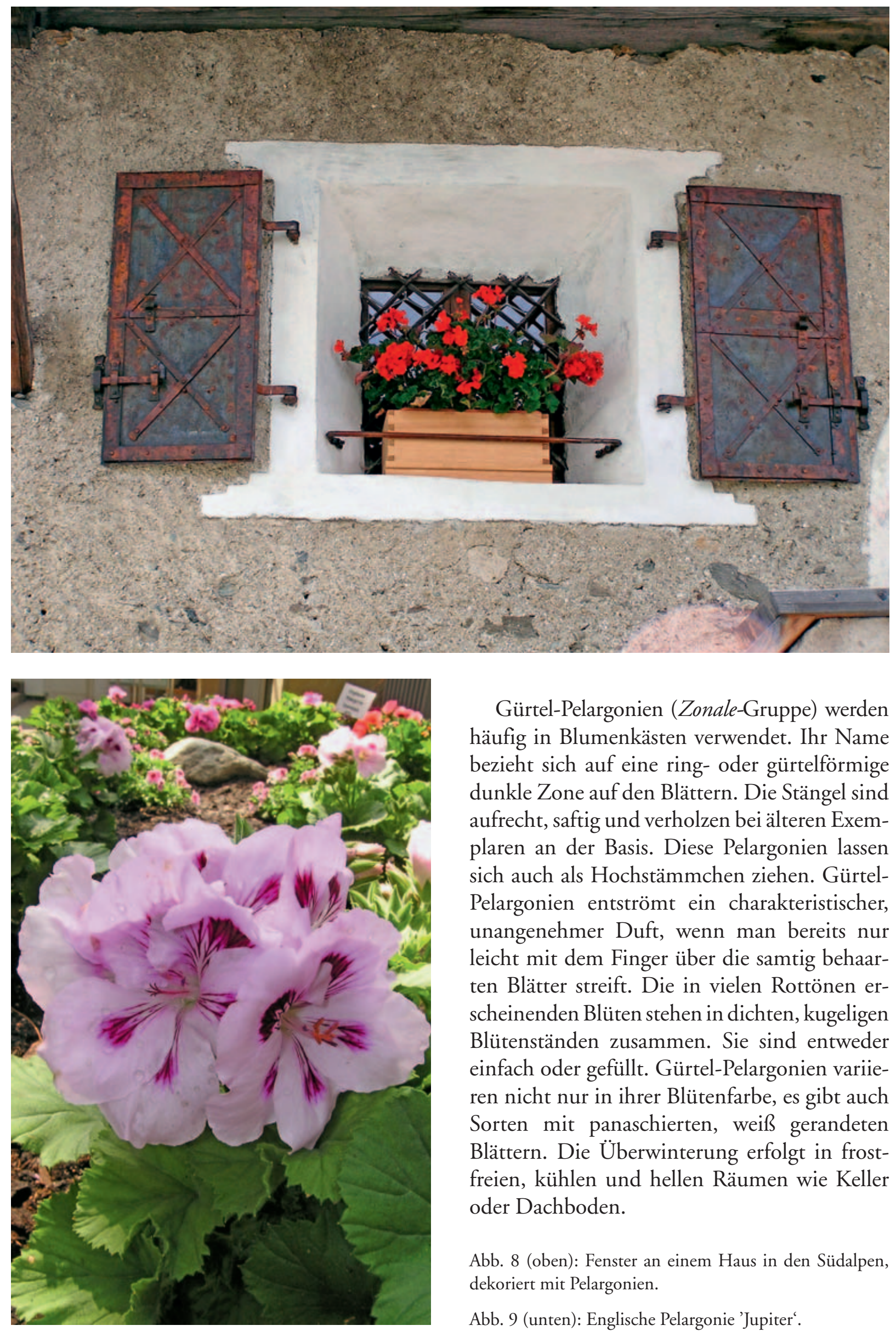

Gürtel-Pelargonien (Zonale-Gruppe) werden häufig in Blumenkästen verwendet. Ihr Name bezieht sich auf eine ring- oder gürtelförmige dunkle Zone auf den Blättern. Die Stängel sind aufrecht, saftig und verholzen bei älteren Exemplaren an der Basis. Diese Pelargonien lassen sich auch als Hochstämmchen ziehen. GürtelPelargonien entströmt ein charakteristischer, unangenehmer Duft, wenn man bereits nur leicht mit dem Finger über die samtig behaarten Blätter streift. Die in vielen Rottönen erscheinenden Blüten stehen in dichten, kugeligen Blütenständen zusammen. Sie sind entweder einfach oder gefüllt. Gürtel-Pelargonien variieren nicht nur in ihrer Blütenfarbe, es gibt auch Sorten mit panaschierten, weiß gerandeten Blättern. Die Überwinterung erfolgt in frostfreien, kühlen und hellen Räumen wie Keller oder Dachboden.

Abb. 8 (oben): Fenster an einem Haus in den Südalpen, dekoriert mit Pelargonien.

Abb. 9 (unten): Englische Pelargonie 'Jupiter'. 
Efeu-Pelargonien (Grandiflorum-Gruppe) eignen sich sehr gut für Balkonkästen und Blumenampeln, weil ihre Triebe hängen oder kriechen. Sie können von April bis zum Herbst im Freien gehalten werden. Ihr deutscher Name bezieht sich auf die efeuähnlichen Blätter. Sie sind dicklich, glatt und glänzend. Während des Wachstums benötigen Efeu-Pelargonien viel Wasser und alle zwei Wochen eine Düngergabe. Überwintert werden sollten sie in einem kühlen trockenen, hellen Raum. Nach der Überwinterung ist es gut, wenn die Erde in den Pflanzgefäßen ausgetauscht wird.

Edel-Pelargonien, Englische Geranien (Grandiflorum-Gruppe) sind vor allem für das Zimmer geeignet. Man sollte sie nach draußen höchstens im Sommer, und dann nur an einen geschützten Platz, z. B. auf Balkon oder Terrasse, stellen. Ihre nierenförmigen, gezähnten Blätter erreichen etwa Handgröße. Von April bis zum Frühherbst erscheinen die in doldigen Ständen angeordnete Blüten. Sie sind rosa, rot oder weiß gefärbt mit dunklen Flecken. Die Pflanzen benötigen feuchte Erde ohne stauende Nässe und einen hellen Standort, aber keine pralle Sonne. Sie können bei etwa $12{ }^{\circ} \mathrm{C}$ überwintert werden, wobei hier ein helles, kühles Gewächshaus oder Zimmer deutlich besser geeignet sind als ein Keller.

\section{Duftpelargonien}

Hierunter versteht man überwiegend Pelargonien, deren Blätter einen charakteristischen Duft z. B. nach Rose, Apfel, Minze, Erdbeere, Kampfer oder Zitrone aufweisen. Die Bezeichnungen Pelargonium odoratissimum oder $P$ x fragrans lassen auf die Bedeutung als Duftpflanzen schließen. Die Blüten der Duftpelargonien sind meist kleiner als jene der Edel- und GürtelPelargonien und die Blätter sind je nach Art ganzrandig oder leicht gezähnt bis tief gelappt. Der Duft entwickelt sich am besten, wenn die Pflanzen an einem sonnigen, windstillen Ort wachsen. Im Herbst müssen sie ins Haus geholt und kühl und trocken überwintert werden. Dies kann nach einem kräftigen Rückschnitt z. B. im Keller erfolgen.

Abb. 10: Gürtel-Pelargonie 'Vectis Glitter'.

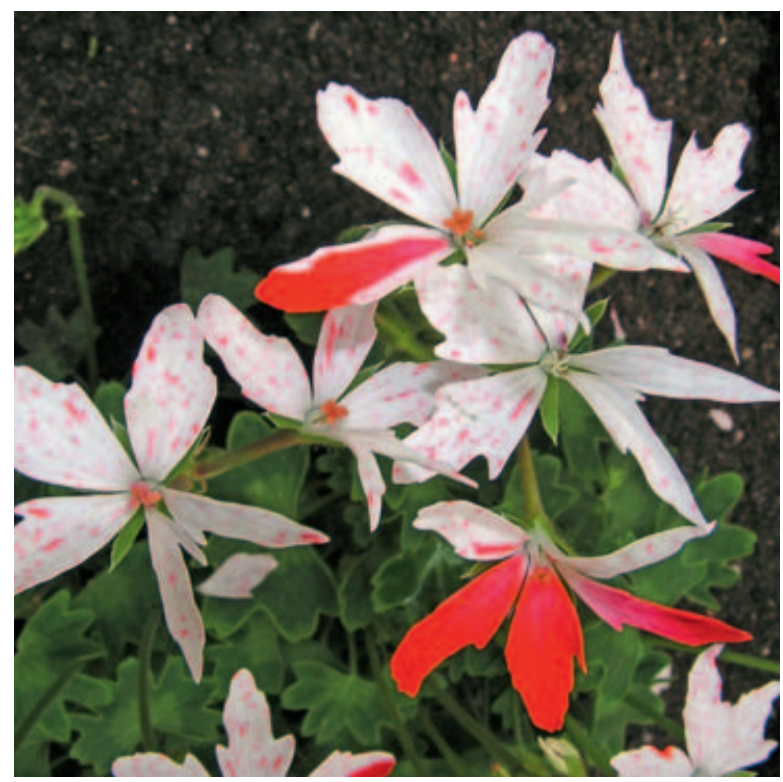

Die drei wichtigsten Pelargonien, die zur Gewinnung des aromatischen Geraniumöls verwendet werden, sind $P$. graveolens, $P$. radens und $P$. capitatum. Besonders P. graveolens ist sehr variabel und Stammmutter vieler Duftpelargonien. Es gibt u.a. Varietäten mit behaarten Blättern und Minzduft oder mit rauen Blättern, die nach Rosen duften. Die Vermehrung erfolgt leicht mittels Stecklingen. Damit bleibt bei den daraus hervorgehenden Klonen der typische aromatische Duft der Mutterpflanze erhalten.

Duftpelargonien werden besonders in Nordafrika, auf Madagaskar, Réunion, in Frankreich, China und Brasilien zur Duftstoffgewinnung angebaut. Verwendung finden vor allem die Blätter, die während der Blütezeit geerntet und anschließend einer Wasserdampfdestillation unterzogen werden. Für die Herstellung von einem Liter Geraniumöl werden 80 kg Pelargonienblätter benötigt. Geraniumöl findet in der Kosmetik und Nahrungsmittelindustrie Verwendung. Auch in der Aromatherapie wird es gegen Gürtelrose, Schuppenflechte und Neurodermitis eingesetzt.

Aus getrockneten Pelargonienblättern lassen sich ganz einfach Duftbeutelchen herstellen. Bevor man die zerkleinerten Blätter in die Säckchen gibt, kann man sie mit Irispulver bestreuen, weil sich dadurch der Duft länger hält und Motten abgeschreckt werden. Der Duft der Pelargonien kann auch in der Küche verwendet 

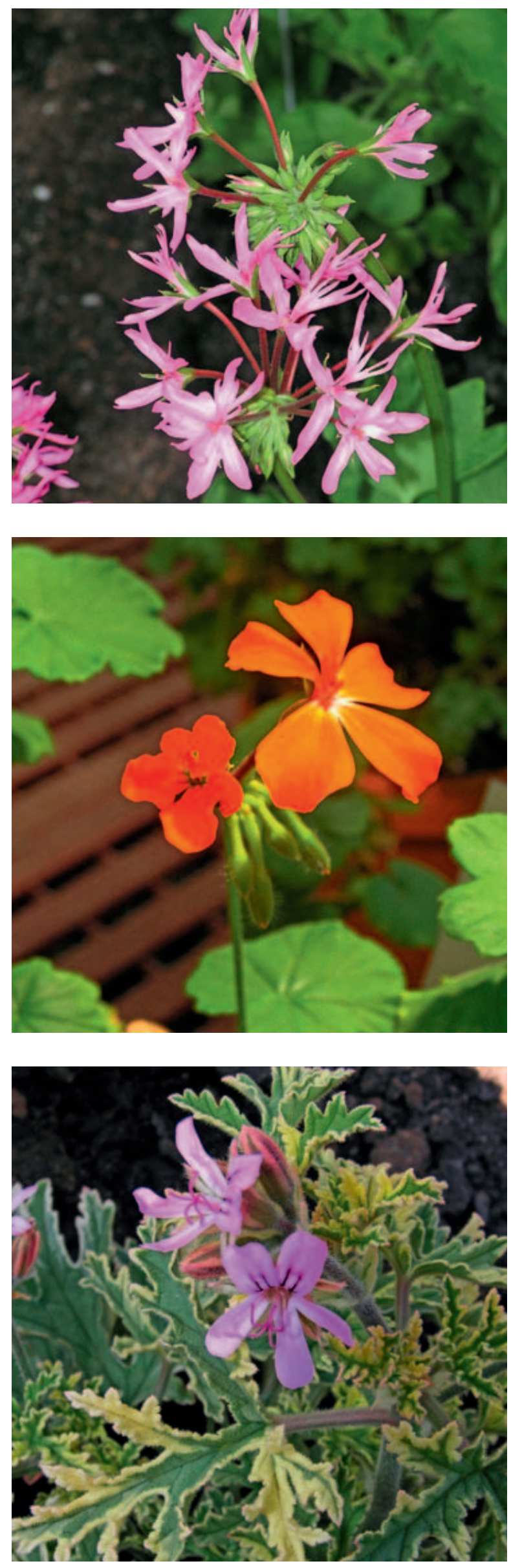

werden. In England beispielsweise aromatisiert man traditionell schwarzen Tee, indem Pelargonienblätter zusammen mit dem Teepulver in eine Dose gelegt werden. In Kärnten werden Blätter des Rosenkrautes (P. radens) in den Hochzeitsstrauß gebunden oder dem Bräutigam in das Knopfloch gesteckt.

\section{Storchschnabel und Pelargonium als Heil- pflanzen}

In der Volksmedizin spielen verschiedene Storchschnabelgewächse eine Rolle. Bereits gemäß der mittelalterlichen Signaturlehre, nach der anhand des äußeren Erscheinungsbildes der Pflanzen auf deren Wirkung geschlossen werden konnte, galt besonders der Blutrote Storchschnabel ( $G e$ ranium sanguineum) als blutstillendes Mittel, weil sich seine Blätter im Herbst blutrot verfärben. Kraut und Wurzeln enthalten u. a. Gerbstoffe und wurden zur Wundbehandlung sowie bei Geschwüren eingesetzt. Der Stinkende Storchschnabel ( $G$. robertianum) wurde früher auch wegen seiner Heilwirkung Gottesgnadenkraut genannt und gegen eiternde Wunden verwendet. Der in Nordamerika beheimatete Storchschnabel ( $G$. maculatum) soll Brechreiz bei Magengeschwüren lindern.

Einige Pelargonien finden in der südafrikanischen Volksmedizin Verwendung, worauf bereits der Artname Pelargonium antidysentericum schließen lässt. Die fleischigen Knollen dieser Art werden bei Durchfallerkrankungen in Milch gekocht getrunken, direkt gekaut, in Pulverform Speisen zugegeben oder als Abkochung verwendet. Pelargonium betulinum ist eine bis $1,3 \mathrm{~m}$ hohe Pflanze mit kahlen bis leicht behaarten Zweigen. Die Blätter erinnern an Birkenblätter. Ihr deutscher Name Kampferblatt bezieht sich auf den kampferartigen Duft zerriebener Blätter. Die Pflanze wurde früher ähnlich wie Kampfer-

Abb. 11 (oben): Manche Pelargonien wie hier die Sorte 'Laura Rita' haben sternförmige Blüten.

Abb. 12 (Mitte): Pelargonium inquinans. Im Vergleich mit den Kultursorten sind die Blüten von Wildarten relativ klein.

Abb. 13 (unten): Pelargonium graveolens, eine klassische Duftpelargonie. 
pulver gegen Husten verwendet. Pelargonium pulverulentum hat eine große, unterirdische Knolle mit zerrissener Borke. Ihre blaugrünen Blätter sind etwas sukkulent und mehlig behaart. Der behaarte Blütenstand kann bis $50 \mathrm{~cm}$ hoch werden. Im 19. Jh. glaubte man, durch das Richten der Knolle auf Feinde deren Waffen ungefährlich machen zu können. Die Wurzeln wurden zudem gegen Durchfall und Ruhr eingenommen, während Blätter als Mittel gegen Hämorrhoiden galten. Pelargonium luridum ist durch eingeschnittene Blätter und rosa Blüten gekennzeichnet. Zulus verwenden die Wurzeln gegen Durchfall. Getrocknete und zermahlene Wurzeln werden von ihnen in Brei gemischt gegen Ruhr eingenommen.

Umckaloabo ist ein in Deutschland angebotenes Medikament aus einem Extrakt der verdickten Wurzeln von Pelargonium reniforme bzw. P. sidoides. Der Produktname stammt aus der Zulu-Sprache und bedeutet „schwerer Husten". Das nicht rezeptpflichtige Arzneimittel wird als pflanzliches Antibiotikum bei akuten und chronischen Infektionen insbesondere der Atemwege verwendet und es soll das Immunsystem stärken. Jährlich werden über 4 Millionen Packungen im Wert von mehr als 50 Mio. $€$ (Apothekenverkaufspreis) verkauft. Ende des 19. Jh. wurden in England Pelargonium-Extrakte gegen Tuberkulose eingesetzt. Details zur Wirksamkeit müssen noch erforscht werden.

Pelargonien sind also eine attraktive, zugleich z. T. medizinisch wirksame Pflanzengruppe. Es ist lohnenswert, sich mit Sorten- und Artenvielfalt zu befassen und vielleicht auch den eigenen Balkon oder Garten mit ihnen zu bestücken.

\section{Dank}

Für kritische Durchsicht des Manuskriptes und fachliche Ergänzungen danke ich FranK SCHUMACHER (Botanischer Garten der Universität Wien).

Abb. 14 (oben): Geranium sanguineum galt lange als wichtige Heilpflanze innerhalb der Storchschnabelgewächse.

Abb. 15 (unten): Pelargonium sidoides.
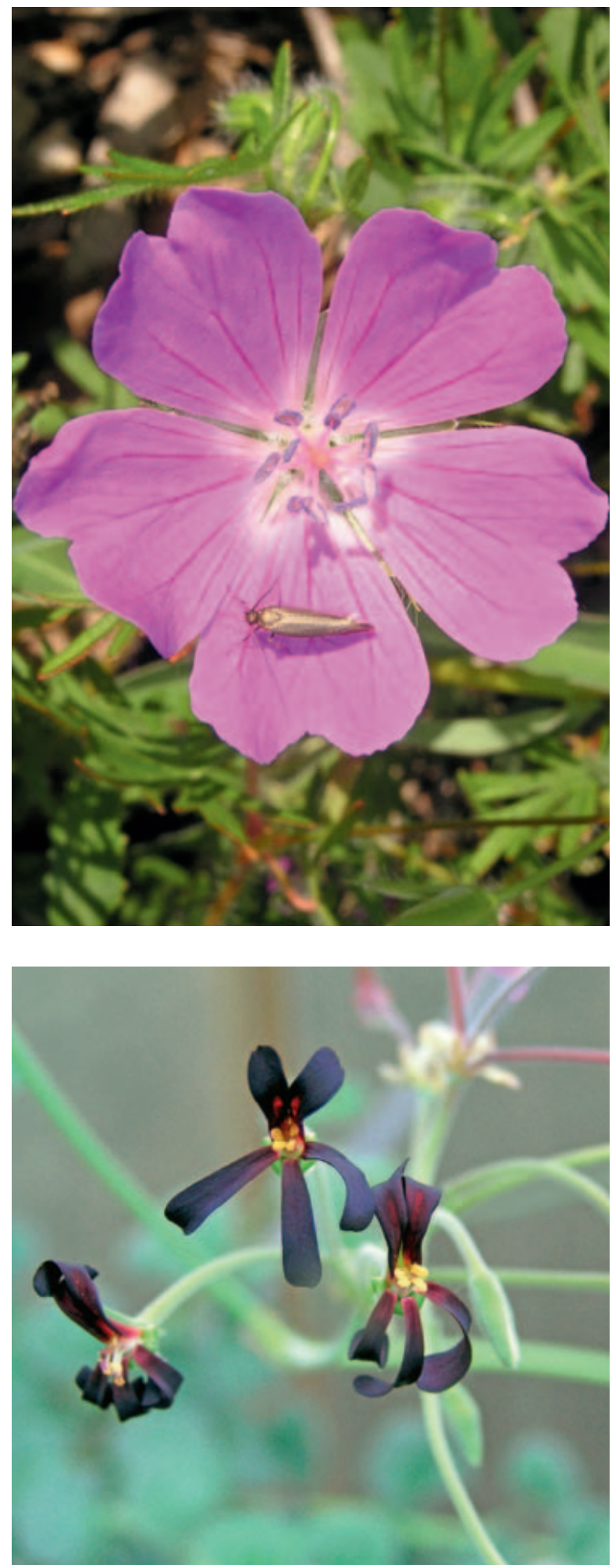

Literatur

Gugenham, E. 1981: Geranien und Pelargonien.Stuttgart.

van der Walt, J. J. A. 1979: Pelargonien des südlichen Afrika. Bd. 1-3. - Limburg.

Wiegele, M. 2000: Duftpelargonien. - Stuttgart.

YeO, P. F. 1985: Geranium. - Stuttgart. 